Research article

\title{
Gaze position interferes in body sway in young adults
}

\author{
Carolina Menezes Fiorelli ${ }^{\mathrm{a}, \mathrm{b}}$, Paula Fávaro Polastri ${ }^{\mathrm{b}, \mathrm{c}}$, Sérgio Tosi Rodrigues ${ }^{\mathrm{b}, \mathrm{c}}$, \\ André Macari Baptista ${ }^{c}$, Tiago Penedo ${ }^{c}$, Vinicius Alota Ignácio Pereira ${ }^{c}$, Lucas Simieli ${ }^{c}$, \\ Fabio Augusto Barbieri ${ }^{\mathrm{b}, \mathrm{c}, *}$
}

a Universidade Sagrado Coração, Department of Physical Therapy, Health's Sciences Center, Bauru, SP, Brazil

b São Paulo State University (Unesp), School of Sciences, Laboratory of Information, Vision and Action (LIVIA), Department of Physical Education, Bauru, SP, Brazil

c SãoPaulo State University (Unesp), School of Sciences, Human Movement Research Laboratory (MOVIE-LAB), Department of Physical Education, Bauru, SP, Brazil

\section{A R T I C L E I N F O}

\section{Keywords:}

Quiet stance

Postural control

Eye movements

Motor control

Eye fixation

Gaze position

\begin{abstract}
A B S T R A C T
Postural control is influenced by eye movements. Gaze fixation, which comprises a component of ocular vergence, is important in the acquisition of highly specific task information, but its relation to postural control is little investigated. The aim of the study was to investigate the effects of gaze fixation position (central and lateral fixations) on postural sway in young adults. Forty young adults with ages ranging from 20 to 35 years were invited to participate in the study. Postural sway was measured in quiet stance in bipedal support in three 60-s trials under the following conditions: gaze fixation on a target positioned in front of participant, gaze fixation on a target positioned on right side of participant, and gaze fixation on a target positioned on left side of participant. The following center of pressure parameters (COP) in the anteroposterior (AP) and mediolateral directions (ML) were analyzed for each of the trials: body sway displacement, mean velocity of sway, root mean square (RMS) of sway, and median frequency. In addition, detrended fluctuation analysis (DFA) exponent, in anteroposterior and medio-lateral directions, was calculated. The COP presented greater AP and ML displacement ( $\mathrm{p}<0.03$, effect size $=1.37$; and $\mathrm{p}<0.03$, effect size $=1.64$, respectively) and RMS AP and ML $(\mathrm{p}<0.04$, effect size $=1.66$; and $\mathrm{p}<0.02$, effect size $=2.50$, respectively) for lateral gaze fixation compared to central gaze fixation. These results suggest that gaze fixation on a laterally positioned target increases body sway in anteroposterior and mediolateral directions.
\end{abstract}

\section{Introduction}

Postural control ensures guidance and necessary stability in all tasks performed [1] and visual information plays an important role in the generation of postural synergies for postural control during bipedal posture [2]. Periods of relative gaze stability (fixations) allow the acquisition of relevant information of the scene, providing highly specific layout of the environment [3]. The time of gaze fixations, gaze position, and sequence in which gaze fixations occur are closely linked to the task and are controlled by cognitive systems $[3,4]$. In sports and many daily activities, it is necessary for humans to fixate objects that are not positioned in front of them, for example, when typing on a computer and redirecting the gaze alternately between the keyboard and the monitor (vertical movement) or between the keyboard and a document positioned on the side of the keyboard (horizontal movement).

When people fixate their gaze on a target, rotational movements of the eye are responsible for redirecting their position to the side, regardless of whether the head or neck have been moved, and also for stabilizing the movement of the head [5]. Gaze fixation on an object occurs through the simultaneous movement of both eyes [6], and this coordination is maintained by the synergic action of the eye muscles [7]. Distinct areas of the brain stem mediate the commands coming from the cerebral cortex to the reticular formation, and then to the nuclei of the cranial nerves responsible for conjugate eye movements (oculomotor, trochlear, and abducens nerves) [6]. Due to spontaneous body sway, the image of the environment moves on the retina; this retinal slip (directly related to these oscillations) is used by the central nervous system as feedback for compensatory sway [8]. Therefore, vision can facilitate stabilization of upright posture, by enabling detection of self-motion relative to structures in the visual field [9].

Body sway is influenced by visual information. How body sway is influenced by the direction of the gaze movement in saccades [10-13], smooth pursuit eye movements [10], and ocular vergence that must occur in fixations [13-15] have been previously studied. Stoffregen and

\footnotetext{
* Corresponding author at: São Paulo State University (Unesp) - Campus Bauru, Human Movement Research Laboratory MOVIE-LAB, Department of Physical Education, School of Sciences, Av. Luiz Edmundo Carrijo Coube, 14-01, Vargem Limpa, CEP: 17033-360, Bauru, SP, Brazil.

E-mail addresses: cmenezesfiorelli@yahoo.com.br (C.M. Fiorelli), barbieri@fc.unesp.br (F.A. Barbieri).
} 
colleagues [17] found that postural sway is lower when directing the gaze at an oscillatory target than at a stationary target. Horizontal saccades and pursuit eye movements also seem to attenuate body sway when compared with gaze fixation $[10,11,17]$. When we move the eyes compared to when we look forward, the proprioceptive or afferent signals of the extraocular muscles are activated, which provides input to the postural system to improve stability [14]. In addition, Kapoula and Lee [14] showed that in young adults, at a distance of $200 \mathrm{~cm}$, body sway is reduced when there is elevation or depression of the eye relative to the frontal fixation, indicating an improvement in postural control. However, at a shorter distance $(40 \mathrm{~cm})$, the eye-gaze position did not affect body sway relative to the frontal fixation. Thus, the combination of these findings seems to indicate that gaze fixation on an object (target) positioned laterally could also attenuate body sway. However, to date, no studies have investigated the effects of gaze fixation, without head movement, on a target positioned laterally to the individual, on body sway. Previous studies have investigated the influence of gaze lateral fixation on postural control, but the individuals oriented their head towards one of target directions $[13,15]$ or performed a difficult free-searching task without gaze fixation in one target [16].

Within this context, we intend to answer the following question: does lateral gaze fixation attenuate body sway? The aim of the study was to investigate the effects of gaze fixation position (frontal and lateral fixations) on postural sway in young adults. The hypothesis was that lateral gaze fixation would reduce body sway in young adults, corroborating previous studies that manipulated the gaze fixation position [14] and analyzed the saccadic lateral movement [11,17].

\section{Method}

\subsection{Participants}

Forty young adults with ages ranging from 20 to 35 years were invited to participate in the study. The following exclusion criteria were established for participation in the study: impairment in visual acuity even with the use of corrective lenses (values greater than 20/20 in the Snellen test) [18], the use of drugs that interfere in postural control, the presence of musculoskeletal and/or neuromuscular impairments in the previous 6 months, and balance disorders that would prevent performance of the required tasks. All participants were blinded to the purposes of the experiment. Prior to experimental procedures, participants signed a written consent form approved by the local ethics committee. All study procedures were performed in accordance with the ethical standards laid down in the Helsinki Declaration.

Seven participants were excluded from the initial sample due to the following exclusion criteria: one participant reported a sprained ankle in the previous three months, one participant presented impairment in visual acuity (20/40 on the Snellen test), and four participants were excluded due to problems in data collection. Thus, the sample of this study included 33 participants ( 21 men, age $-22.30 \pm 3.20$ years; height $-1.71 \pm 0.08 \mathrm{~m}$; body mass $-70.90 \pm 10.40 \mathrm{~kg}$ ).

\subsection{Procedures}

The participants came to the laboratory on one-single day. First, they responded to an interview to confirm that they did not meet the exclusion criteria of the study. Subsequently, the Snellen test was used to check visual acuity. Finally, body sway was assessed.

Postural sway was evaluated during quiet standing in bipedal stance and the participants were barefoot. The feet were positioned side-byside, hip width apart [19]. To ensure that the participants maintained their feet in the same position in all trials a kraft paper sheet was used, the size of the force platform, on which the contour of each participant's feet was drawn.

Each participant performed three 60-s trials under the following conditions: gaze fixation on a target positioned in front of participant,

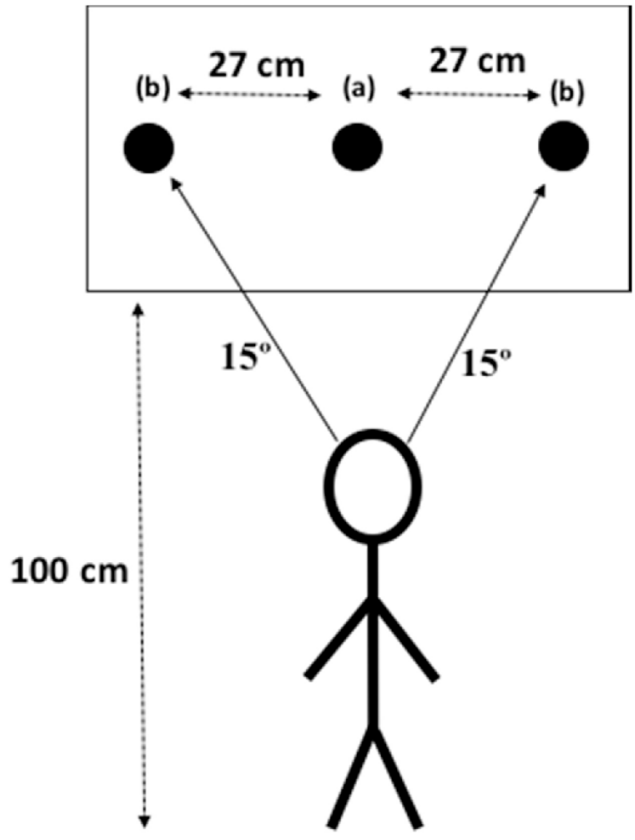

Fig. 1. Schematic representation of the central target position (a) and lateral target positions (b).

gaze fixation on a target positioned on right side of participant and gaze fixation on a target positioned on left side of participant. Each participant performed a total of 9 trials. Each block of trials for each condition was randomized for each participant. The participants were instructed to maintain an upright standing position, with head positioned forward and without moving, and gaze directed at a target positioned according to the condition held. The study was conducted in an isolated room that had no other visual information apart from the target.

For the frontal gaze fixation task (frontal target), the target was positioned $100 \mathrm{~cm}$ away from the participant. For the lateral gaze fixation task, the target was positioned $27 \mathrm{~cm}$ from the frontal target, requiring a $15^{\circ}$ visual angle (Fig. 1 ). This angle was used to allow participants to fix one point without moving the head [17]. During the tasks, only the target that corresponded to the condition was kept visible to the participant, avoiding any distractions. Furthermore, one researcher observed and verified each participant's appropriate gaze fixation on the target using a small camera (Microsoft webcam, model 1407, $60 \mathrm{~Hz}$ ). After data collection, videos were analyzed to reconfirm the required gaze fixation for each condition. When, during data collection, the participants did not keep their eye fixated on the target, or performed any body movement, the trial was discarded and performed again.

\subsection{Data collection and analysis}

Body sway of the participants was measured using a force plate (AccuGait, Advanced Mechanical Technologies, Boston, MA), $50 \mathrm{~cm} \times 50 \mathrm{~cm}$, with a sample rate of $120 \mathrm{~Hz}$. The resultant CoP signal of the ground reaction force was determined in a 2-dimensional transverse plane by means of digital moment-of-force calculations. The first $10 \mathrm{~s}$ of each recording were ignored to avoid potential disturbances resulting from delayed stabilization after the participant stepped onto the force plate. The data were filtered with a fourth order low-pass Butterworth filter with cutoff frequency of $5 \mathrm{~Hz}$. The following parameters of $\mathrm{CoP}$ in anteroposterior and medio-lateral directions were calculated for each trial: displacement (the length of the CoP trajectory on the support base); mean velocity (the displacement of the total sway of the CoP divided by the total duration of the trial); the root mean square (RMS - the CoP variability around the mean CoP trajectory) 
and median frequency of sway. The last parameter was calculated by employing spectral analysis of the position time series separately in each direction (Matlab software version 7.10, Mathworks). In addition, detrended fluctuation analysis (DFA) exponent, in anteroposterior and medio-lateral directions, was calculated $[20,21]$. DFA exponents vary between 0 and 2 . When DFA $<0.5$ or $1<$ DFA $<1.5$, the signal is anti-persistent (smaller DFA $=$ more anti-persistent). When $0.5<$ DFA $<1$ or $1.5<$ DFA $<2$, the signal is persistent (larger DFA $=$ more persistent). Some diversity exists regarding how these exponents are to be practically interpreted [21].

During postural control data collection, participants used the Head Tracker system (EYE HEAD ${ }^{\mathrm{TM}}$ integration) to verify possible head movements. The sample rate was $60 \mathrm{~Hz}$. The Head Tracker system consists of a magnetic transmitter to track head position. The device uses a fixed point as a reference and software calculates the distance between this fixed point and the Head Tracker. To verify the position of the head, the average position of the head in the anteroposterior, lateral, and longitudinal axes were calculated for each trial and analyzed separately for each axis of motion.

\subsection{Statistical analysis}

The significance level was set at 0.05 for all analyzes and SPSS 18.0 (SPSS, Inc.) was used for statistical analysis. The Shapiro-Wilk and Levene's tests were employed to check the normal distribution of data and homogeneity of variances, respectively. The average of the right and left trials was calculated. The parameters of the CoP and head position were analyzed by ANOVA with repeated measures for condition (central gaze fixation $x$ lateral gaze fixation). The effect size (etasquared) was calculated for each statistical analysis and interpreted as small (effect size $<0.2$ ), medium (effect size between 0.3-0.7), or large (effect size $>0.8$ ) [22].

\section{Results}

As expected, there was no significant difference in head position in the anteroposterior, lateral or longitudinal axes $(\mathrm{F} 1,32=0.15$, $\mathrm{p}=0.70 ; \mathrm{F} 1,32=0.11, \mathrm{p}=0.73 ; \mathrm{F} 1,32=0.01, \mathrm{p}=0.89$, respectively) between the central and lateral gaze fixation conditions. The CoP (Fig. 2) of the participants demonstrated greater anteroposterior and mediolateral displacement $(\mathrm{F} 1,31=4.77, \mathrm{p}<0.03$, effect size $=1.37$; and $\mathrm{F} 1,31=4.76, \mathrm{p}<0.03$, effect size $=1.64$, respectively) and RMS (F1,31 $=4.22, \mathrm{p}<0.04$, effect size $=1.66$; and $\mathrm{F} 1,31=5.36, \mathrm{p}<0.02$, effect size $=2.50$, respectively) for the lateral gaze fixation condition compared to the central gaze fixation condition. There were no significant effects of gaze fixation for mean velocity, median frequency of sway and DFA exponent in anteroposterior and mediolateral directions $(\mathrm{p}>0.05)$.

\section{Discussion}

The main question of the study was whether lateral gaze fixation attenuates body sway. Our results seem to indicate that lateral gaze fixation did not attenuate body sway. Fixating the gaze on a laterally positioned target seems to increase the anteroposterior and mediolateral body sway compared to fixating the gaze on a centrally positioned target (forward gaze fixation). There were effects on anteroposterior and mediolateral displacement and RMS, but without effects on other parameters. The increased anteroposterior displacement was contrary to previous findings for horizontal saccades [17], which indicated an increase in body sway only in the mediolateral direction. We can interpret this difference in findings as a consequence of the task since horizontal saccades induce mediolateral adjustments while fixation of the gaze on a lateral position did not seem to produce this effect. In addition, the absence of effects on other parameters, such as body sway velocity and DFA, seems to indicate a functional strategy to perform the lateral fixation efficiently. The participants need to adjust body sway displacement and its variability, but without changing temporal parameters. Thus, the results of our study contradict our hypothesis that lateral gaze fixation reduces body sway in young adults. Three alternative mechanisms could be considered to explain the higher body sway during the lateral gaze fixation.

First, increased postural sway during lateral gaze fixation seems to be a postural control strategy to deal with the task. To obey an instruction given to the participants prior to the task, "keep your eyes fixated on the target and don't move your head," and to adjust the system to possible fluctuations in optical flow due to the position of the eye, the participants appeared to be successful in the gaze fixation task (since none of the participants moved their head or stopped gaze fixation on the lateral target) in a manner detrimental to postural control, increasing body sway. The increased postural sway during lateral gaze fixation suggests that despite visual and postural behaviours presenting a strong synergy in the control, which may require a bidirectional feedforward command with postural and visual control mutually modulated with respect to each other [23]. The visual system seems to lead the feedforward control of postural sway due to the instruction given in the task. On the other hand, the postural task performed by young adults in this study is quite easy, which allows an increase in body sway. However, if we think about a sport or daily situation that requires fine postural control when we fixate the gaze on a side object, a small change in maintaining postural stability (i.e., higher body sway) could decrease performance in the task or result in a fall. In the same view, the postural system can tolerate more instability when the target is further away. In the present study, the target was more distant in the lateral conditions $(100 \mathrm{~cm}$ with the target in the center and $104 \mathrm{~cm}$ with the target in the lateral position); there is evidence that greater (frontally positioned) target distance by itself increases body sway $[24,25]$.

Second, gaze fixation on an object for 60-s is not a task normally performed by individuals, which could cause fatigue of the extraocular muscles. Under normal conditions, when the head can move freely or saccadic movements can be performed, the individual combines moments of gaze fixation with eye saccadic or head movements, allowing the individual to require less effort from extraocular muscles and keep their eyes on the center of the orbit [5]. In addition, when the eye is in the primary position, the majority of the fatigue-resistant units of the orbital and global layers are recruited for the fine and sustained gradation of force needed for fixation and binocular vision [26]. However, when the eyes move laterally, there is an increase in muscular tension to keep the eyes in this position [27]. Collins and collaborators [27] verified that levels of tension required to maintain fixation at each position of gaze vary from a minimum of $8-12 \mathrm{~g}$ approximately $15^{\circ}$ outside the muscle's field of action to a maximum of around $40 \mathrm{~g}$ at extreme gaze within the muscle's field of action. Furthermore, the lateral fixation task of the present study is not usually performed by the participants, which could result in fatigue shortly after the beginning of the task. Thus, maintaining gaze fixation at a point (object) laterally positioned for the time required in the present study may cause fatigue of the eye muscles that perform the lateral gaze fixation, possibly affecting postural control.

Third, fixation on a laterally positioned object, with the head oriented forward, increases the divergence of the eyes to focus on the target. By stabilizing the gaze sideways, as mentioned above, the distance from the target to the individual increased by $4 \mathrm{~cm}$. To fixate the target sideways without moving the head to the side, participants had to increase the eye divergence. When individuals fixate a lateral target and move their head to the target, eye divergence does not happen. Different from our findings, this no-divergence task did not cause effects of gaze orientation on body oscillation when a target was fixated laterally [15]. According to Kapoula and Lee [14] gaze divergence occurs when we fixate an object that is farther away without head movement. In addition, the authors indicate that the oculomotor 

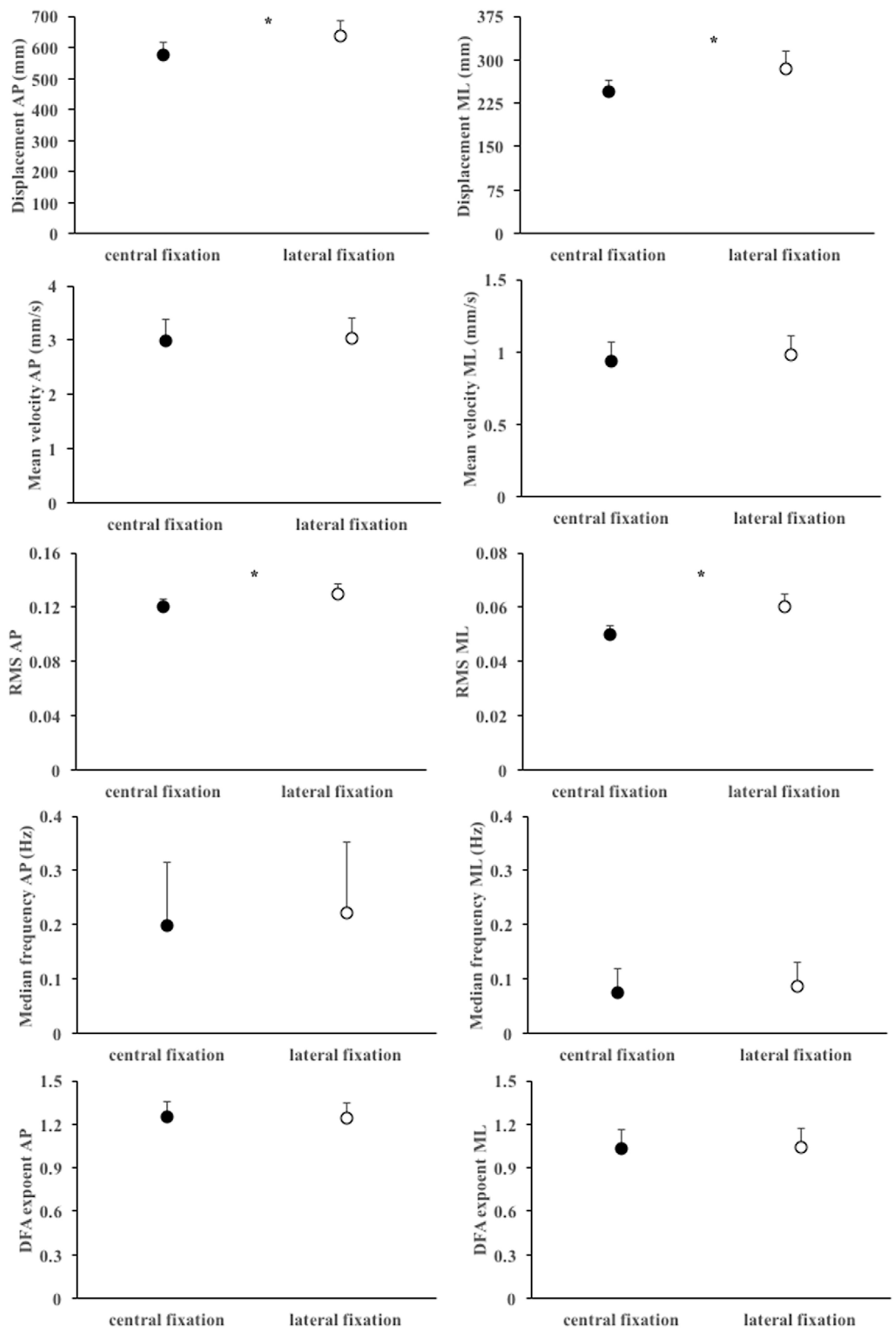

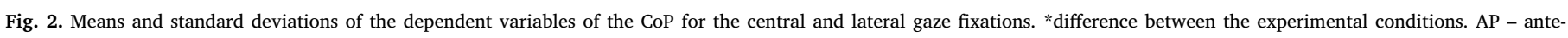
roposterior; ML - mediolateral.

convergence that occurs when viewing closer objects promotes postural stability in the quiet standing posture, decreasing body sway, since proprioceptive information of extraocular muscles that influence postural stability are improved. Finally, extraocular proprioception contributes to the eye, head, and body position coding in relation to postural and environmental conditions [28,29]. 


\section{Conclusion}

In conclusion, gaze fixation on a laterally positioned target increases body sway in anteroposterior and mediolateral directions. These results confirm the influence of gaze position on postural stability which should be considered when performing tasks requiring attentional focus and postural stability simultaneously, for example, sports such as archery.

\section{References}

[1] A. Shumway-Cook, M.H. Woollacott, Motor Control: Translating Research into Clinical Practice, Lippincott Williams \& Wilkins, 2007.

[2] A. Danna-Dos-Santos, A.M. Degani, T.W. Boonstra, L. Mochizuki, A.M. Harney, M.M. Schmeckpeper, C.T. Leonard, The influence of visual information on multimuscle control during quiet stance: a spectral analysis approach, Exp. Brain Res. 233 (2015) 657-669.

[3] M. Hayhoe, D. Ballard, Eye movements in natural behavior, Trends Cogn. Sci. 9 (2005) 188-194.

[4] J.M. Henderson, Human gaze control during real-world scene perception, Trends Cogn. Sci 7 (2013) 498-504.

[5] E.R. Kandel, J.H. Schwartz, T.M. Jessell, Principles of Neural Science, fourth ed., McGraw-Hill, New York, 2000.

[6] C. Rashbass, G. Westheimer, Independence of conjugate and disjunctive eye movements, J. Physiol. 159 (1961) 361-364.

[7] L. Lundy-Ekman, Neuroscience: Fundamentals for Rehabilitation, fourth ed., Saunders, St. Louis, 2013.

[8] M. Guerraz, A.M. Bronstein, Ocular versus extraocular control of posture and equilibrium, Clin. Neurophysiol. 38 (2008) 391-398.

[9] J.D. Tübingen, T. Brandt, Visual-vestibular interaction: effects on self-motion perception and postural control, in: R. Held, H.W. Leibowitz, H. Teuber (Eds.), Perception, Springer, Heidelberg, Berlin, 1978, pp. 755-804.

[10] S.T. Rodrigues, P.F. Polastri, J.C. Carvalho, J.A. Barella, R. Moraes, F.A. Barbieri, Saccadic and smooth pursuit eye movements attenuate postural sway similarly, Neurosci. Lett. 584 (2015) 292-295.

[11] S.T. Rodrigues, S.A. Aguiar, P.F. Polastri, D. Godoi, R. Moraes, J.A. Barela, Effects of saccadic eye movements on postural control stabilization, Motriz 19 (2013) 614-619.
[12] F. Rey, T.T. Le, R. Bertin, Z. Kapoula, Saccades horizontal or vertical at near or at far do not deteriorate postural control, Auris Nasus Larynx 35 (2008) 185-191.

[13] C.T. Bonnet, C. Morio, S. Szaffarczyk, P.R. Rougier, Postural mechanisms to contro body displacements in the performance of lateral gaze shifts, J. Motor Behav. 46 (2014) 97-405.

[14] Z. Kapoula, T.T. Le, Effects of distance and gaze position on postural stability in young and old subjects, Exp. Brain Res. 173 (2006) 438-445.

[15] Y.P. Ivanenko, R. Grasso, F. Lacquaniti, Effect of gaze on postural responses to neck proprioceptive and vestibular stimulation in humans, J. Physiol. 15 (1999) 301-314.

[16] C.T. Bonnet, S. Szaffarczyk, S. Baudry, Functional synergy between postural and visual behaviors when performing a difficult precise visual task in upright stance, Cogn. Sci. 41 (6) (2016) 1675-1693.

[17] T.A. Stoffregen, B.G. Bardy, C.T. Bonnet, R.J. Pagulayan, Postural stabilization of visually guided eye movements, Ecol. Psychol. 18 (2006) 191-222.

[18] M. Yanoff, J.S. Duker, Ophthalmology, fourth ed., Elsevier Health Sciences, 2013.

[19] N. Termoz, S.E. Halliday, D.A. Winter, J.S. Frank, A.E. Patla, F. Prince, The control of upright stance in young, elderly and persons with Parkinson's disease, Gait Posture 27 (2008) 463-470.

[20] D. Lin, H. Seol, M.A. Nussbaum, M.L. Madigan, Reliability of COP-based postural sway measures and age-related differences, Gait Posture 28 (2008) 337-342.

[21] J.A. Norris, A.P. Marsh, I.J. Smith, R.I. Kohut, M.E. Miller, Ability of staticand statistical mechanics posturographic measures to distinguishbetween age and fall risk, J. Biomech. 38 (2005) 1263-1272.

[22] J. Cohen, Statistical Power Analysis for the Behavioral Sciences, second ed., Routledge, Abingdon, 1988.

[23] C.T. Bonnet, S. Baudry, A functional synergistic model to explain posturalcontrol during precise visual tasks, Gait Posture 50 (2016) 120-125.

[24] S.T. Rodrigues, J.G. Jardim, N.S. Siqueira, P.F. Polastri, S.A. Aguiar, Postural stabilization of looking: effects of object distance, Braz. J. Motor Behav. 6 (2011) 7-15.

[25] T.A. Stoffregen, L.J. Smart, B.G. Bardy, R.J. Pagulayan, Postural stabilization of looking, J. Exp. Psychol. Hum. Percept. Perform. 25 (1999) 1641-1658.

[26] G. Lennerstrand, Strabismus and eye muscle function, Acta Ophthalmol. 85 (2007) 711-723.

[27] C.C. Collins, D. O'Meara, A.B. Scott, Muscle tension during unrestrained human eye movements, J. Physiol. 245 (1975) 351-369.

[28] K. Jahn, M. Strupp, S. Krafczyk, O. Schüler, S. Glasauer, T. Brandt, Suppression of eye movements improves balance, Brain 125 (2002) 2005-2011.

[29] J.P. Roll, J.P. Vedel, R. Roll, Eye, head and skeletal muscle spindle feedback in the elaboration of body references, Prog. Brain Res. 80 (1989) 113-123. 